\title{
Study of the Existence of Global Attractors for the Wezewska, Czyewska and Lasota Models
}

\author{
Edgar Alí Medina ${ }^{1}$, Manuel Vicente Centeno-Romero² ${ }^{2}$ Fernando José Marval López², \\ José Feliciano Lockiby Aguirre ${ }^{3}$
}

${ }^{1}$ Departamento de Ciencias, Universidad de Oriente Núcleo de Nueva Esparta, Guatamare, Venezuela

${ }^{2}$ Departamento de Matemáticas, Escuela de Ciencias, Universidad de Oriente Núcleo de Sucre, Cumaná, Venezuela

${ }^{3}$ Departamento de Informática, Escuela de Ciencias, Universidad de Oriente Núcleo de Sucre, Cumaná, Venezuela

Email address:

edgaralim@gmail.com (Edgar A. M.), manuelcenteno11@gmail.com (M. V. Centeno-Romero),

fmarval59@hotmail.com (Fernando J. M. L.), jlockiby@gmail.com (José F. L. A.)

\section{To cite this article:}

Edgar Alí Medina, Manuel Vicente Centeno-Romero, Fernando José Marval López, José Feliciano Lockiby Aguirre. Study of the Existence of Global Attractors for the Wezewska, Czyewska and Lasota Models. Mathematics Letters. Vol. 7, No. 3, 2021, pp. 37-40.

doi: $10.11648 / \mathrm{j} . \mathrm{ml} .20210703 .11$

Received: May 8, 2021; Accepted: July 21, 2021; Published: August 31, 2021

\begin{abstract}
In this research we present a study of global attractors in mathematical models of differential equations, which are an important tool in mathematics; furthermore, taking advantage of the stability of the solutions, it was possible to determine the control of biomedical phenomena, among other aspects, present in various population groups. Likewise, differential equation models are used to simulate biological, epidemiological and medical phenomena, among others. The reference population groups used in this research are the family of population models given by the differential equations $N^{\prime}(t)=p(t, N(t))$ - $\mathrm{d}(\mathrm{t}, \mathrm{N}(\mathrm{t})$ ). A particular case of this family of differential equations is the mathematical model called the Wezewska, Czyewska and Lasota (WCL) model, whose form is given by: $N^{\prime}(t)=p e^{(-q)}-\mu N(t)$. This model describes the survival of red blood cells (erythrocytes) in humans. The WCL model, in discrete variable, has a non-trivial global attractor. In this research we demonstrate, using the Schwarz derivative technique, the existence of at least one model global attractor. On the other hand, the results of the present investigation showed the existence of a single fixed point, as the only global attractor characterized by the equation $\mathrm{N}=\mathrm{pe}^{(-\mathrm{qN})}-\mu \mathrm{N}$.
\end{abstract}

Keywords: Global Attractor, Point Balances, Differential Equations, Fixed Point

\section{Introduction}

The study of global attractors in models of differential equations, as well as their existence, definition, stability and application in various spaces and models, has been treated in recent years by several researchers, as expressed in [1-8]. Similarly, fractional Lasota-Wazewska models have been studied in $[9,10]$.

This article is motivated by works [1-3], where the authors study the existence of global attractors for equations in differences of the Wezewska, Czyewska and Lasota (WCL) model, on the existence of the fixed point. The authors, in the previous works, up to that date, have not found a rigorous test, they only present a graph that gives an idea of the existence, and the uniqueness is not proven, which is an open problem. It should be noted that models such as those treated in the works mentioned above were described, for the first time, using differential equations, in [11, 12]. Similarly, epidemiological studies have been carried out using differential equation models, such as those discussed in [13], as well as in [14].

In this research, we consider the family of population models given by:

$$
N^{\prime}(t)=p(t, N(t))-d(t, N(t))
$$

Different biological and medical phenomena were described by differential equations between the 1950s and 1980 s, such as the production of blood cells and the general approach, proposed by equation (1), to describe the dynamics of cell production [15]. In equation (1), p ( $t, N(t))$ is the 
production rate of the cells at time $t$, provided that the population size is $\mathrm{N}$ and $\mathrm{d}(\mathrm{t}, \mathrm{N}(\mathrm{t}))$ is the rate of mortality. It was a common belief for researchers that mortality was proportional to the number of circulating blood cells; that is: $\mathrm{d}(\mathrm{t}, \mathrm{N}(\mathrm{t}))=\mu \mathrm{N}(\mathrm{t})$. However, in this research we consider $\mathrm{p}(\mathrm{t}$, $\mathrm{N}(\mathrm{t}))=\mathrm{pe}^{(-\mathrm{qN}(\mathrm{t}))}$ and the model

$$
N^{\prime}(t)=p e^{-q N(t)}-\mu N(t)
$$

to prove the existence of a single fixed point, as a single global attractor, characterized by equation (2).

\section{Methods}

A documentary research was carried out with a qualitative approach; Since a study of global attractors in models of differential equations is presented, these tools of great importance in mathematics. Behavior and responses are observed, by collecting all possible bibliographic material on the subject, making a detailed study of it. The hypotheticaldeductive method, characteristic of mathematical research, was used, so that the conjectures could be answered during the development of the work, which led to the achievement of the objectives. The present work shows an applied form that confronts theory with reality $[17,18]$.

\section{Preliminaries}

Consider the system of differential equations

$$
y^{\prime}(t)=f(y(t), \text { con } t>0,
$$

where $f$ is a map of $C^{\infty}(I)$, with $I=[a, b]$. Let's consider the linearized system:

$$
y^{\prime}(t)=\left[\frac{\partial f}{\partial x}(x *)\right] y(t)
$$

Where $x^{*}$ is an equilibrium point of equation (4); that is, $f(x *)=0$.

Definition 1. An equilibrium point or stationary solution of a differential equation is a solution $y(x)=a$ (constant) for all $x$ $\in R$. That is, the stationary solutions or equilibrium points are those whose graphs are straight horizontal.

Definition 2 . The equilibrium point $\mathrm{x} *$ is locally stable for system (4), if for each $\varepsilon>0$, there exists $\delta>0$, such that if $\left|y_{o}-x^{*}\right|<\delta$, then $\left|y\left(t, t_{0}, y_{0}\right)-x\left(t, t_{0}, x^{*}\right)\right|=$ $\left|y\left(t, t_{0}, y_{0}\right)-x^{*}\right|<\varepsilon$.

Definition 3. We will say that equation (3) has a fixed point at $c$, if $f(c)=c$.

Definition 4. Let $f: \Omega \subseteq R^{n} \rightarrow R^{n}$ be a $C^{1}$ field and let $x_{0}$ be an equilibrium point of $x^{\prime}=f(x)$.

1. It is said that $x_{0}$ is stable, if for all $\varepsilon>0$ there exists $\delta>$ 0 , such that for all $x \in B\left(x_{0}, \delta\right) \Phi x(t)$ is defined for all $t \geq 0$ and we have $\Phi x(t) \in B\left(x_{0}, \varepsilon\right)$ for all $t \geq 0$.

2. It is said that $x_{0}$ is asymptotically stable, if $x_{0}$ is stable and there is also $r>0$, such that $\lim _{t \rightarrow \infty} \Phi x(t)=x_{0}$, for all $x \in B\left(x_{0}, r\right)$.

Definition 5. An equilibrium point $x^{*}$ is globally asymptotically stable, if it is locally stable and $\operatorname{alsolim}_{t \rightarrow \infty} y(t)=x^{*}$

Theorem 1. The real part of the eigenvalue $\left(\partial \mathrm{f} / \partial \mathrm{x}\left(\mathrm{x}^{\wedge *}\right)\right)$ is negative if and only if $x^{*}$ is local asymptotically stable.

Definition 6 . The critical points $\bar{x}$ or the fixed points care locally stable or globally asymptotically stable, if they verify the previous definitions.

Remember that the critical points $\bar{x}$ are those where $f$ $'(\bar{x})=0$.

Definition 7. A function $f: R \rightarrow R$ is contractive, if there is a constant $k<1$ such that for any $x_{1}, x_{2} \in R$ holds:

$$
\mathrm{d}\left(\mathrm{f}\left(\mathrm{x}_{1}\right), \mathrm{f}\left(\mathrm{x}_{2}\right)\right)<\mathrm{k} \cdot \mathrm{d}\left(\mathrm{x}_{1}, \mathrm{x}_{2}\right)
$$

That is, a contractive application is one that contracts the distances with a contraction ratio strictly less than unity. Where, in the set of real numbers, the distance between two of them is defined as follows:

$$
\mathrm{d}\left(\mathrm{x}_{1}, \mathrm{x}_{2}\right)=\left|\mathrm{x}_{1}-\mathrm{x}_{2}\right|
$$

So the definition of contractive application is as follows:

$$
\left|\mathrm{f}\left(\mathrm{x}_{1}\right)-\mathrm{f}\left(\mathrm{x}_{2}\right)\right|<\mathrm{k} .\left|\mathrm{x}_{1}-\mathrm{x}_{2}\right|
$$

Theorem 2 (Contraction). We will say that the map $T: E \rightarrow$ $E, E \subset R$ is a contraction or a contractive operator, if there exists a constant $L \in[0,1)$, such that $|T(x)-T(y)| \leq$ $L|x-y|$, for all $x, y \in E$.

Theorem 3. (Contractive fixed point). If $T: E \rightarrow E$ is a contraction, then $T$ has an unique fixed point on $\mathrm{E}$.

Definition 8 (Derived from Schwarz). The Schwarz derivative is defined for a real function $f$ of class $C^{3}$, as:

$$
(s f)(x)=\frac{f^{\prime \prime \prime}(x)}{f(x)}-\frac{3}{2}\left[\frac{f^{\prime \prime}(x)}{f^{\prime}(x)}\right]^{2}
$$

At all points where $f^{\prime}(0) \neq 0$.

This derivative has been used in differential delay equations with unimodal feedback, verifying that they do not satisfy the Schwarz derivative condition [16].

Theorem 4. (Schwarz's theorem). Let $\mathrm{f}$ of class $C^{3}[a, b]$, with $f:[a, b] \rightarrow[a, b]$, suppose that $f$ is strictly decreasing or with one unique critical point. If $f$ has an unique fixed point at $c$ y $(s f)(x)$ is negative for all $x \neq x^{*}$, where $x^{*}$ is an unique critical point, then the local asymptotic stability condition $0<\left|f^{\prime}(c)\right|<1$, implies the global asymptotic stability for $c$.

\section{Equilibrium Point Analysis}

Theorem 5. The equation $N^{\prime}(t)=p e^{-q N(t)}-\mu N(t)$, with $\mathrm{p}, \mathrm{q} \in[\mathrm{o}, \mathrm{a}]$.

a) If $\mu>0$, it has an unique critical point given by the equation $p e^{-q N}-\mu N=0$.

b) If $-1<\mu<0$, it has no critical points

Theorem 6 . Let $x^{*}$ be the unique equilibrium point of the system $N^{\prime}(t)=p e^{-q N(t)}-\mu N(t)$; that is, $p e^{-p x^{*}}-\mu x^{*}=$ $0(6)$, then $x^{*}$ is local asymptotically stable.

Proof. Calculate the characteristic polynomial relative to $\left[T^{\prime}\left(x^{*}\right)\right]$, obtaining $\lambda=-\left(p q e^{-q x^{*}}+\mu\right)<0$; that is, $x^{*}$ is 
local asymptotically stable.

Theorem 7. If $\mu>0$, the unique equilibrium point of the equation $p e^{. q x^{*}}-\mu x^{*}=0$ (7) is globally asymptotically stable.

Proof. If $\mu>0$, then by Theorem 5 equation (3) has an unique critical point given by the equation $p e^{-q N}-\mu N=0$, let this be $x^{*}$, and by Theorem $6 x^{*}$ is local asymptotically stable.

\section{Fixed Points Analysis}

If $-1<\mu<0$, then the model $N^{\prime}(t)=p e^{-q N(t)}-\mu N(t)$ has an unique critical point given by:

$$
\begin{gathered}
N^{*}=\frac{1}{q} \ln \left(-\frac{p q}{\mu}\right)>0 \\
\text { follows: } T(N)=p e^{-q N}-\mu N, \text { note that } \\
|T(x)-T(y)| \leq p\left|\sum_{n=0}^{+\infty} \frac{1}{n !}(-q x)^{n}-(-q y)^{n}\right|+\mu|x-y| \leq\left(p \frac{1}{a}\left(\sum_{n=0}^{+\infty} q^{n} a^{n}\right)+\mu\right)|x-y| .
\end{gathered}
$$

We take

$$
L=\mu+\frac{1}{a} p\left(\sum_{n=0}^{+\infty} q^{n} a^{n}\right)<1 .
$$

Then $\mathrm{T}$ has an unique fixed point. So, the equation $N^{\prime}(t)=p e^{-q N(t)}-\mu N(t),-1<\mu<0$ has an unique fixed point.

\section{Global Analysis of the Asymptotic Stability of the Fixed Point $C$}

Theorem 9. For $p, q \in(0,1)$ and $-1<\mu<0$, the fixed point $C$ for the equation $N^{\prime}(t)=p e^{-q N(t)}-\mu N(t)$ is global asymptotically stable.

Proof. Let $T(N)=p e^{-q N}-\mu N$, defined in $[0, \mathrm{a}]$, then $T^{\prime}(N)=-p q e^{-q N}-\mu \quad, \quad$ with $0<|T(N)|=$ $\left|q p e^{-q N}+\mu\right|<q p e^{-q N}<1$; that is, for $N=C, 0<\left|T^{\prime}(C)\right|<$ 1 which implies that $C$ is local asymptotically stable. On the other hand,

$$
(s f)(x)=-\frac{p q^{3}\left[p q-2 \mu e^{q x}\right]}{2\left[e^{q x} \mu+p q\right]^{2}}<0
$$

$x^{*}=\frac{1}{q} \ln \left(-\frac{p q}{\mu}\right)>0$, where $\mathrm{x}^{*}$ is the critical point and $(s f)$ $(x)<0, \forall x \neq x^{*}$. Therefore, $C$ is globally asymptotically stable.

\section{Conclusions}

A numerical representation of the solutions of the equation $N^{\prime}(t)=p e^{-q N(t)}-\mu N(t),-1<\mu<0$ it is: $N(n)=$ $\sum_{k=1}^{n}(-\mu)^{n-1+k} p e^{-q N(k)}, \quad$ this series when $\lim _{n \rightarrow+\infty}|N(n)| \rightarrow L$ is convergent and converges to the fixed point $\mathrm{C}$, if $0<p|\mu|<1$. The way to find this sum is by using the recurrence method considering the difference equation $N(n+1)=p e^{-q N(n)}-\mu N(n),-1<\mu<0$.

We note that equation (2) is a scalar differential equation.
Theorem 8. Suppose that the number series:

$$
\sum_{n=0}^{\infty} a^{n-1} q^{n}=\frac{1}{a} \sum_{n=0}^{\infty} a^{n} q^{n}
$$

is convergent and further suppose that:

$$
\left(\mu+\frac{1}{a} p \sum_{n=0}^{\infty} a^{n} q^{n}\right)=L<1,
$$

then the equation $N^{\prime}(t)=p e^{-q N(t)}-\mu N(t),-1<\mu<0$ has an unique fixed point, given by the equation $p e^{-q N}-\mu N=$ $N$.

Proof. Let us define the operator $T:[o, a] \rightarrow[0, a]$, as
It is natural to establish a control in the parameters that are exhibited in the equation in order to guarantee existence of: fixed points, equilibrium points and critical points. The first two are those that, in general, are of extreme importance for the global study of stability. As well as, the technique used in these systems is what we know as the famous Schwarz derivative technique.

For $p, q \in(0,1)$ and $-1<\mu<0$, the fixed point $C$ for the equation $N^{\prime}(t)=p e^{-q N(t)}-\mu N(t)$ is global asymptotically stable.

An interesting open problem would be to pose an analogous model in $\mathrm{R}^{2}$ as follows:

$$
\left\{\begin{array}{l}
x^{\prime}(t)=p_{1} e^{\left(-q_{1} x(t)\right)}-\mu_{1} x(t) \\
y^{\prime}(t)=p_{2} e^{\left(-q_{2} y(t)\right)}-\mu_{2} y(t)
\end{array}\right.
$$

Here it is notable that the system involves more parameters and it is obvious that a control can be established in the parameters to guarantee the fixed points and the equilibrium points. Known techniques to be used will be Dulac, Poincare-Bendixon.

For three-dimensional differential equations the best known technique is the Liapunov functions.

For dimensions in complicated spaces (normed, Banach, among others), more hypotheses must be added in the problem that support the stock conditions fixed points and critical points, and therefore their stability in the global sense.

Another important aspect for differential equations of the abstract type is to be able to establish a definition of generalized dichotomy, which deduces generalized global stability for spaces of the abstract type. These are problems of interest raised in [14].

\section{References}

[1] Wazewska-Czyzewska, M. and Lasota, A. 1976. Mathematical problems of the dynamics of the red blood cells system. Annals of the Polish Mathematical Society Series III. Applied Mathematics 17: 23-40. 
[2] Medina, E. A. 2013. Equivalencia asintótica relativa de ecuaciones en diferencia. Trabajo de Ascenso para optar a la Categoría de Profesor Titular UDO. Venezuela.

[3] Braverman, E. and Saker, S. H. 2011. On a Difference Equation with Exponentially Decreasing Nonlinearity. Hindawi Publishing Corporation Discrete Dynamics in Nature and Society 2011, Article ID 147926, 17 pages doi: $10.1155 / 2011 / 147926$

[4] Bonotto, E. M., Bortolan, M. C., Carvalho, A. N. and Czaja, R 2015. Global attractors for impulsive dynamical systems-a precompact approach. Journal of Differential Equations. 259 (7): $2602-2625$.

[5] Chi, P., Yuncheng, Y. and Jianzhong, S. 2019. Global attractors for Hindmarsh. Rose equations in Neurodynamics. Cornell University. Mathematics, Analysis of PDEs: 1907. 13225.

[6] Lee, J., Nguyen, N. and Toi, Vu M. 2020. Gromov-Hausdorff stability of global attractors of reaction diffusion equations under perturbations of the domain. 269 (1): 125-147.

[7] Yirong, J., Nanjing, H. and Nanjing, H. 2020. American Institute of Mathematical Sciences. 2584: 1193-1212.

[8] Rangel, G. 2019. Global Attractors in Partial Differential Equations. CNRS et Universit'e de Paris-Sud Analyse Num'erique et EDP, UMR 8628. B`atiment 425. F-91405 Orsay Cedex, France. Genevieve.

[9] Li, Y., Wang, Y. and Li, B. 2020. Existence and finite-time stability of a unique almost periodic positive solutionfor fractional-order Lasota-Wazewska red blood cell models. International Journal of Biomathematics. 13 (2).

[10] Stamov, G. and Stamova, I. 2019. Impulsive Delayed LasotaWazewska Fractional Models: Global Stability of Integral Manifolds. Mathematics 7, 1025; doi: 10.3390.
[11] Losson, J., Mackey, M. C. and Longtin, A. 1993. Solution multistability in first-order nonlinear differential delay equations. Chaos 3 (2): 167-176.

[12] Mackey, M. C. and Glass, L. 1977. Oscillation and chaos in physiological control systems. Science 197 (4300): 287-289.

[13] Medina, E. A., Centeno-Romero, M. V. y Marval, F. J. 2019. Global stability of critical points for type SIS epidemiological model. International Journal of Theoretical and Applied Mathematics 5 (6): 94-99.

[14] Medina, E. A. 2016. Equivalencia Asintótica Relativa de Ecuaciones en Diferencia. Tesis Doctoral. Universidad Central de Venezuela. Caracas. Venezuela.

[15] Rigalli, A., Aguirre C., Armendáriz, M. y Cassiraga, G. 2003. Formulación de modelos matemáticos de fenómenos biológicos. Doctorado en Ciencias Biomédicas, Facultad de Ciencias Médicas. Rosario, Argentina.

[16] Franco, D., Guiver, Ch. Logemann, H. and Perán, J. 2020. On the global attractors of delay differential equations with unimodal feedback not satisfyins the negative schwarzian derivatie condition. Electronic Journal of Qualitative Theory of Differential equations. (76): 1-15.

[17] Farji-Brener, A. G. 2007. Una forma alternativa para la enseñanza del método hipotético-deductivo. INCI 32 (10) ISSN 0378-1844.

[18] Tamayo y Tamayo, M. 2011. El Proceso de la Investigación Científica. Editorial Limusa, S. A. de C. V. Grupo Noriega Editores. México. 